\title{
Determinantes sociales y prevalencia de la caries dental en población escolar de zonas rurales y urbanas de Ecuador
}

\author{
Social determinants and prevalence of dental caries in the \\ school population in rural and urban areas of Ecuador
}

Recibido: 2018/06/15. Aceptado: 2018/08/17. Publicado: 2018/09/01

\section{Fernando Ortega Pérez \\ Andrea Guerrero ${ }^{2}$ \\ Paulina Aliaga ${ }^{3}$}

1 Universidad San Francisco de Quito, Colegio de Ciencias de la Salud, Escuela de Salud Pública, Campus Cumbayá, oficina CT 109. Quito. Ecuador.

Correo electrónico: fortega@usfq.edu.ec

2 Universidad San Francisco de Quito, Colegio de Ciencias de la Salud, Escuela de Odontología, Clínica Odontológica, Campus Cumbayá, oficina CO 106. Quito. Ecuador.

Correo electrónico: odandreaguerrero@gmail.com

${ }^{3}$ Universidad San Francisco de Quito, Colegio de Ciencias de la Salud, Escuela de Odontología, Decanato, Campus Cumbayá, oficina CO 106. Quito. Ecuador.

Correo electrónico: paliaga@usfq.edu.ec 


\section{Resumen}

La caries dental obedece a múltiples factores causales y constituye un problema de salud pública local y global. objetivo: explorar la asociación entre determinantes sociales y prevalencia de caries dental en población escolar rural y urbana en Ecuador. Materiales y Método: Estudio descriptivo de corte transversal realizado por un equipo odontológico adiestrado bajo los criterios de la OMS, que realizó un examen clínico oral a 518 menores de 3 a 15 años y lo complemento con información sobre hábitos higiénico/alimentarios, acceso a servicios de salud y condiciones sociales mediante una encuesta sociodemográfica previamente validada. Resultados: La presencia de caries en dientes definitivos se asocia significativamente con: caries en dientes primarios $p<.000$, ausencia de cepillo dental en la escuela $p<.003$, falta de acceso a una unidad de salud oral $p<.006$, y baja autopercepción de salud oral $p<.012$. La caries en dientes primarios se asocia significativamente con: sexo masculino $p<.001$, baja autopercepción de salud oral $p<.013$, última visita al dentista en más de seis meses $p<.040$, y ausencia de cepillo en la escuela $p<.050$. Conclusión: Se confirma la influencia de múltiples factores causales en la presencia de caries en dientes primarios y definitivos de la población escolar estudiada.

Palabras clave: determinantes sociales, salud oral, prevalencia de caries dental, índice CPOD, población escolar.

\section{Abstract}

Dental caries is a consequence of multiple causal factors and constitutes a local and global public health problem. Objective: to explore the association between social determinants and prevalence of dental caries in school-age population of rural and urban areas in Ecuador. Materials and Methods: Cross sectional study developed by a dental team trained under the World Health Organization criteria applied an oral clinical examination of 518 children aged 3 to 15 years and also complemented data collection with a socio-demographic survey for information regarding hygienic and dietary habits, access to health services and social conditions of children. Results: Caries in definitive teeth is statistically associated to: caries in primary teeth $p<.000$, absence of toothbrush in school $p<.003$, lack of access to an oral health unit $p<.006$, and poor self-perception of oral health $p<.012$, and. Caries in primary teeth is significantly associated to: male sex $p<.001$, poor self-perception of oral health $p<.013$, last visit to the dentist in more than six months $p<.040$, and absence of toothbrush in schook $<.050$. Conclusion: Among the participating school age children of this study, dental caries is a consequence of multiple causal factors.

Keywords: social determinants, oral health, prevalence of dental caries, MDFT score, school population. 


\section{Introducción}

Hace décadas se reconoce a nivel global que la enfermedad bucodental más frecuente es la caries dental, su alta prevalencia constituye preocupación especial para las autoridades de salud pública del mundo como la Organización Mundial de la Salud 1. La pérdida de unidades dentarias no es sólo consecuencia del envejecimiento, sino más bien, de un proceso que se inicia tempranamente en la vida y se incrementa bajo condiciones higiénicas, alimentarias, ambientales y económicas desfavorables.

Varios autores como Curtis 2017, Berton 2017, Hong Ha 2015, Verdugo 2010, reconocen como factores determinantes de caries dental al tipo de agua consumida, la frecuencia de cepillado dental diario, el consumo frecuente de hidratos de carbono, especialmente los dulces, la situación económica de la familia, la accesibilidad cultural, física y económica a las unidades de salud y la aplicación de programas de salud especialmente dirigidos a su prevención, como son la fluorización del agua o de la sal y el tamizaje odontológico periódico.

América Latina es un continente socialmente diverso y por tanto poseedor de una problemática de salud variada que obedece a procesos sociohistóricos que han generado amplias desigualdades. La naturaleza multifactorial de la caries dental puede ser el reflejo de dichas condiciones dispares donde distintos estilos y modos de vida, variados micro nichos ecológicos, condiciones socioeconómicas adversas y disimiles modelos de atención de la salud, podrían convertirse, entre otros, en los factores determinantes de un estado de salud oral especifico.

La naturaleza multifactorial de la caries dental demanda un diagnóstico complejo y procedimientos preventivos y curativos apropiados. Los efectos de la caries no son únicamente el dolor y la potencial pérdida de la unidad dental afectada sino también efectos psicológicos en el individuo y su familia, alteración en la función masticatoria y digestiva, ausentismo en labores cotidianas, y disminución de la calidad de vida.

En el informe sobre enfermedades bucodentales, la Organización Mundial de la Salud (WHO 2004), no desestimó en señalar a los principales determinantes socioculturales de la salud, como: pobreza, bajo nivel de instrucción y falta de tradición que fomente la higiene bucodental. Por otra parte, propuso a los países que velen por un adecuado uso del flúor en la prevención de la caries dental, la dotación de agua y el fomento de prácticas de higiene oral.

\section{Materiales y métodos}

Se trata de un estudio descriptivo de corte transversal aplicado en población preescolar y escolar distribuida en tres regiones ecológicas distintas del Ecuador: Toachi-Pilaton en la zona rural húmeda tropical (800 msnm); barrios La Comuna y Patronato San Jose del centro colonial y Santos Pamba del área periurbana de Quito en la zona interandina (2800 msnm); y en la comunidad rural alto andina de Cangahua (3600 msnm).

Tomando en consideración que todas las comunidades solicitaron la presencia de las Brigadas Odontológicas - USFQ (trabajo de vinculación con la comunidad), los criterios de inclusión consistieron en brindar la atención a todos los niños y niñas que dieron su asentimiento oral y cuyos padres habían conferido su consentimiento informado a las autoridades escolares. En esos niños, se procedió a aplicar los cuatro procedimientos de la brigada: 1) el cuestionario (previamente validado) de datos socio-demográficos (nombres y dos apellidos, edad, sexo, lugar de residencia, nivel de instrucción, acceso a servicios de salud, ocupación de los padres, tipo de agua y servicio higiénico en casa, condición mórbida en el último 
mes, autopercepción de la salud oral, última visita al dentista, concepto de caries y placa bacteriana, conocimiento sobre los alimentos potencialmente generadores de caries, frecuencia de cepillado, frecuencia diaria de ingesta de dulces y bebidas azucaradas, cepillo personal en casa y escuela), 2) el diagnóstico de condiciones de salud oral como: placa bacteriana, cálculos, enfermedad periodontal, mala oclusión, gingivitis y fluorosis y el levantamiento de índices ceod y CPOD, basados en el formulario 033 de la historia clínica del Ministerio de Salud del Ecuador, 3) Charla educativa, y 4) Sellantes de fosa y fisuras y fluorización dental.

El diagnóstico clínico fue realizado en las aulas escolares mediante el método visual-táctil con luz natural y baja lenguas descartables. Los datos fueron registrados en fichas individuales. En total 518 niños y niñas cumplieron los criterios de inclusión y recibieron atención odontológica entre septiembre 2017 y marzo 2018.

Para el análisis de los datos se procedió a la revisión de las fichas individuales, limpieza de datos, digitación de los mismos en una matriz Excel, para luego transcribir la información al programa estadístico SPSS versión 19. Se obtuvieron medidas estadísticas descriptivas confeccionando tablas de frecuencias y tablas de contingencia para las variables sociodemográficas y análisis de correlación para las variables del CPOD/ceod por condiciones sociodemográficas.

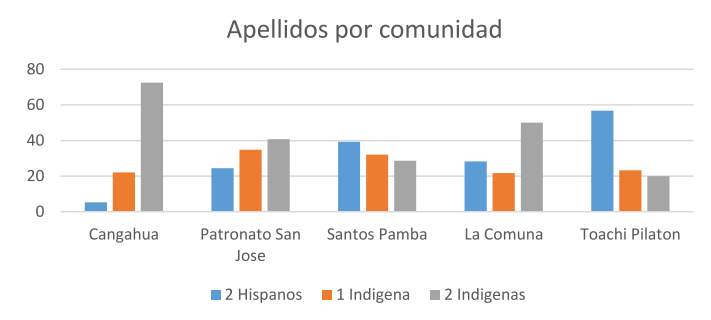

FIGURA \#1. COMPOSICIÓN ÉTNICA ANCESTRAL POR COMUNIDAD.
No tiene acceso Si tiene acceso

\begin{tabular}{|l|l|l|}
\hline Cangahua & 43,5 & 56,6 \\
\hline Patronato San José & 51,2 & 48,8 \\
\hline Santos Pamba & 41,1 & 58,9 \\
\hline La Comuna & 26,1 & 73,9 \\
\hline Toachi Pilatón & 77,3 & 22,7 \\
\hline Promedio & 53,5 & 46,6 \\
\hline
\end{tabular}

\section{CUADRO \#1. ACCESO A SERVICIOS DE SALUD ORAL}

\section{Resulltados socio demográficos}

Se examinó un total de 518 niños y niñas comprendidos entre 3 y 15 años con una media de $9.24 \pm 2.6$ años. El grupo estuvo compuesto por $49.5 \%$ de niñas y $50.5 \%$ de niños. El promedio de años de estudio de educación primaria fue de $5 \pm 2.59$.

Según Instituto Nacional de Estadísticas y Censos, INEC, en los dos últimos censos nacionales de población (2001 y 2010), no más del $7 \%$ de los ecuatorianos se auto identificó como pertenecientes a una nacionalidad indígena. En este estudio hemos procedido a la revisión de los dos apellidos de los niños, para proponer una proxi-variable a la identificación étnica en las comunidades atendidas. Estamos conscientes que el resultado de esta "propuesta" a la interpretación de etnicidad de los ecuatorianos es nada más que una aproximación.

Mediante la aplicación de esta "proxi-variable" se comprobó que $30.9 \%$ de los niños participantes tenía dos apellidos no indígenas, $28.4 \%$ tenía un apellido indígena y otro no indígena, y $40.7 \%$ tenía dos apellidos indígenas. Estos resultados tan solo comprueban la existencia de ancestros indígenas en siete de cada diez niños estudiados y con ello, la posibilidad de patrones tradicionales de alimentación e higiene que en algo podrían diferir de otras comunidades con una composición étnica distinta. Estos resultados no corresponden a la auto identificación étnica de ninguno de los niños participantes. 
Por comunidad, la figura \#1 presenta diversos porcentajes: en Cangahua 94.6\% de niños tenían uno o dos apellidos indígenas, en el Patronato San José $75.5 \%$, en Santos Pamba 60.7\%, en La Comuna $71.7 \%$ y en Toachi-Pilatón $43.3 \%$.

Cangahua 3600 msnm, P San José, Santos Pamba y La Comuna 2800 msnm, Toachi - Pilaton $800 \mathrm{msnm}$

En promedio, 53.5\% de los niños examinados dijo no tener acceso a servicios de salud. La correlación entre caries dental y falta de acceso a servicios de salud tuvo una asociación estadísticamente significativa con la presencia de caries dental (Pearson Chi² 114.860, p<.000).

Las condiciones socioeconómicas de la población fueron estudiadas indirectamente interpretando la actividad económica de los progenitores. Dos tercios de las actividades paternas están relacionados con: Construcción 17.3\%, Servicios 14.6\%, Agricultura 11.2\%, Oficios 9.4\%, Comercio $8.3 \%$ y Guardianía $5.0 \%$. Las ocupaciones que requieren título profesional apenas llegaron a $1.3 \%$. Por otra parte, el $14.5 \%$ de los niños no pudo dar una respuesta respecto del trabajo de sus padres.

Para la ocupación paterna habíamos considerado lo siguiente: Construcción el rol de albañil. Oficios tales como carpintería, plomería, electricista, mecánico, panadero, сегrajero, cocinero. Servicios comprende auxiliar, cargador, estibador, lava carros, mensajero, jornalero, reciclador. Transporte se refiere a taxistas y choferes.

Las principales actividades maternas son: Quehaceres domésticos 33.3\%, actividades comerciales (ventas) 20.2\%, agricultura $19.8 \%$. Actividades que requieren un título profesional apenas alcanzan $1.7 \%$.
Más de la mitad de los niños cree utilizar agua potable en casa $51.5 \%$, seguidos por quienes creen que el agua es únicamente entubada $33.2 \%$. Solamente $12.5 \%$ de niños comentó que el agua es hervida en casa.

Los niños atendidos provienen en su mayoría de zonas rurales, urbano-marginales o barrios populares de Quito y sus respuestas indican el empleo mayoritario del "baño" 90.5\%, seguido por la letrina $5.8 \%$. Persisten prácticas higiénicas de precariedad sanitaria en apenas $3.3 \%$ de casos.

La palabra "Baño" se refiere a un concepto que en el caso de nuestros informantes podría tener como sinónimos a letrina o servicio higiénico. Aquí el hallazgo importante es reconocer que 96.7 \% de niños utilizan estos servicios y no el "campo" o los cultivos, como una alternativa.

En relación con el estado de salud de los niños durante el mes previo a nuestra visita, $84.4 \%$ mencionó haber estado sano, 9.8\% refirió un estado gripal y el restante $5,4 \%$ dijo haber sufrido dolencias menores, como dolor abdominal o fiebre.

La auto percepción de la salud oral demostró que $34.1 \%$ de niños considera tener buena o muy buena salud oral y, por el contrario, 65.9\% dijo tener una condición regular (49.7\%) o mala (15\%).

\begin{tabular}{|l|r|r|}
\hline Variables & Adecuado & No adecuado \\
\hline Última visita al dentista & 27,4 & 72,6 \\
\hline Concepto de caries dental & 40,8 & 59,2 \\
\hline Concepto de placa bacteriana & 12,3 & 87,7 \\
\hline Alimentos productores de caries & 65,9 & 34,1 \\
\hline Como mantener la salud oral & 84,2 & 15,8 \\
\hline Cepilla sus dientes 2 veces o mas & 82,3 & 17,7 \\
\hline Consume dulces 2 veces o mas & 48,3 & 51,7 \\
\hline
\end{tabular}

CUADRO \#2. PORCENTAJE DE CONOCIMIENTOS Y PRÁCTICAS ADECUADAS Y NO ADECUADAS EN SALUD ORAL. 


\begin{tabular}{|c|c|c|c|c|}
\hline Numerador & Denominador & Operación & Índice & SD \\
\hline $\begin{array}{l}\text { Niños con dientes } \\
\text { primarios cariados } \\
254\end{array}$ & \# dientes cariados 905 & $\begin{array}{l}\text { índice c = } \\
905 / 518\end{array}$ & 1.75 & $\begin{array}{r}S D \\
2.48\end{array}$ \\
\hline $\begin{array}{l}\text { Niños con dientes } \\
\text { primarios extraídos } \\
78\end{array}$ & \# dientes extraídos 220 & $\begin{array}{l}\text { índice e = } \\
220 / 518\end{array}$ & 0.42 & $\begin{array}{r}\text { SD } \\
1.48\end{array}$ \\
\hline $\begin{array}{l}\text { Niños con dientes } \\
\text { primarios obturados } \\
136\end{array}$ & \# dientes obturados 283 & $\begin{array}{l}\text { índice o = } \\
283 / 518\end{array}$ & 0.55 & $\begin{array}{r}\text { SD } \\
1.17\end{array}$ \\
\hline Índice ceod & $\begin{array}{l}\text { Número de dientes cariados + } \\
\text { extraídos + obturados / Número de } \\
\text { niños examinados }\end{array}$ & $\begin{array}{l}\text { Índice ceod = } \\
1408 / 518\end{array}$ & 2.71 & $\begin{array}{r}S D \\
3.48\end{array}$ \\
\hline $\begin{array}{l}\text { Nivel de severidad de } \\
\text { caries dental }\end{array}$ & & & MODERADO & \\
\hline
\end{tabular}

\section{CUADRO \#3. CÁLCULO DEL ÍNDICE CEOD}

Respecto de la última visita al dentista, solamente $27.4 \%$ había concurrido en el periodo de los últimos seis meses (recomendación odontológica), 17.3\% lo hizo entre 6 y 12 meses, $34.3 \%$ hace más de un año y $20.2 \%$ nunca visito un dentista.

Demostró tener conocimientos apropiados de lo que significa caries dental el $40.8 \%$ de niños, placa bacteriana $12.3 \%$, alimentos potencialmente productores de caries $65.9 \%$ y qué se debe hacer para mantener una dentadura sana $84.2 \%$ (uso de cepillo y pasta dental), los resultados demuestran una realidad distinta al hábito de cepillado dental. El número promedio de veces diarias que los niños dijeron cepillar sus dientes es de $2.34 \pm$. 86, con una mediana de 3 veces. Solo $2.5 \%$ no cepilla sus dientes, $15.3 \%$ lo hace una vez, $30.1 \%$ dos veces al día y $52.2 \%$ de niños cepilla sus dientes tres o más veces al día.

El número promedio de ocasiones de ingesta de alimentos azucarados como caramelos o bebidas endulzadas es de: $1.87 \pm 1.47$ veces con una mediana de 2 y una moda de 1 .
Del total de 518 niños, $12.5 \%$ no consume dulces, $35.7 \%$ lo hace una vez al día, $26.4 \%$ consume dos veces y $25.3 \%$ consume tres veces o más al día.

Tiene cepillo en casa el $97.3 \%$ de niños, pero en la escuela solo el $44 \%$ lo tiene.

\section{Resultados clínico-odontológicos}

El cuadro \# 3 nos permite reconocer la existencia de un Índice ceod promedio "moderado", cuyo valor alcanza 2.71 .

El cuadro \# 4 nos permite reconocer la existencia de un Índice CPOD promedio de 1.78, en el cual se reconoce un nivel de severidad de caries dental BAJO.

\section{Otras Variables Odontológicas}

El cuadro \# 5 presenta la prevalencia de caries dental en escolares de seis a diez años que alcanza un valor de $37,9 \%$ y una razón promedio de caries en cada niño de 2.28 .

A diferencia de la prevalencia en el grupo de seis a diez años, el cuadro \# 6 presenta la prevalencia 


\begin{tabular}{|c|c|c|c|}
\hline Numerador & Denominador & Operación & Índice \\
\hline $\begin{array}{l}\text { Niños con dientes } \\
\text { definitivos cariados } 246\end{array}$ & \# dientes cariados 705 & $\begin{array}{l}\text { índice } c= \\
705 / 518\end{array}$ & 1.36 \\
\hline $\begin{array}{l}\text { Niños con dientes } \\
\text { definitivos perdidos } 11\end{array}$ & \# dientes perdidos 15 & $\begin{array}{l}\text { índice e = } \\
15 / 518\end{array}$ & 0.02 \\
\hline $\begin{array}{l}\text { Niños con dientes } \\
\text { definitivos obturados } 78\end{array}$ & \# dientes obturados 155 & $\begin{array}{l}\text { índice o = } \\
155 / 518\end{array}$ & 0.29 \\
\hline Índice CPOD & $\begin{array}{l}\text { Número de dientes cariados + extraídos + } \\
\text { obturados / Número de niños examinados }\end{array}$ & $\begin{array}{l}\text { Índice CPOD = } \\
875 / 518\end{array}$ & 1.78 \\
\hline $\begin{array}{l}\text { Nivel de severidad de } \\
\text { caries dental }\end{array}$ & & & BAJO \\
\hline
\end{tabular}

\section{CUADRO \#4. CÁLCULO DEL ÍNDICE CPOD}

de caries dental en escolares de once a quince años cuyo valor es $54.7 \%$, demostrando un incremento en el número de caries a mayor edad, así como una razón promedio de 3.84 caries por niño, es decir un incremento considerable de $68 \%$.

\section{Fluorosis}

Ya que este artículo se ha enfocado en la problemática de la caries dental, incluimos los resultados relacionados con la exposición ambiental al flúor, debido a la condición ecológica en que se disponen territorialmente las colectividades estudiadas.

La fluorosis dental es una condición que afecta el esmalte del diente y es causada por un consumo excesivo de fluoruro durante un extenso periodo mientras los dientes se están formando bajo las encías. La fluorosis compromete la dentición infantil incrementando el riesgo a presentar caries dental.

Al examen físico pudimos constatar 149 casos de niños con fluorosis (37.3\%) y 325 niños libres de fluorosis, es decir $62.7 \%$. Las lesiones leves representan el $28.8 \%$, las moderadas $6.9 \%$ (36 casos) y las severas $1.5 \%$ (8 casos), es decir $0.5 \%$ más que lo comúnmente encontrado en población general. El diagnóstico clínico de fluorosis detectó un incremento paulatino de esta condición conforme aumenta la edad, así, por debajo de los 7 años se encontró en $10.6 \%$ de niños afectados, entre 7 y 12 años se detectó en $37.4 \%$ y finalmente en niños de 13 a 15 años esta condición fue observada en $63.6 \%$. El diagnóstico de fluorosis dental varía entre comunidades, mientras en Cangahua se observó en $64.1 \%$ de estudiantes afectados, en los barrios de Quito el promedio fue de $44.7 \%$ y en Toachi Pilatón fue $6.7 \%$.

\section{Asociación entre las variables sociodemográficas y la prevalencia de caries}

A continuación, se analiza el grado de asociación entre las variables socio demográficas y la presencia de caries en dientes primarios y en dientes definitivos, para realizarlo se ha empleado un análisis de regresión logística, cuyos resultados se expone en los cuadros 7 y 8 con los valores de $\mathrm{Chi}^{2}$ y su correspondiente valor de significación estadística.

La estadística Chi es la diferencia en -2 log-de probabilidad entre el modelo final y el modelo reducido. El modelo reducido se forma omitiendo un efecto del modelo final. 


\begin{tabular}{|l|c|r|}
\hline Prevalencia de caries dental en escolares de seis a diez años & N & $\%$ \\
\hline Total de niños de 6 a 10 años: & 266 & $100 \%$ \\
\hline Total de niños de 6 a 10 años que NO presentaron caries: & 165 & $62 \%$ \\
\hline Total de niños de 6 a 10 años que SI presentaron caries: & 101 & $37.9 \%$ \\
\hline Total de caries en los 101 niños: & 230 & \\
\hline Razón de caries por niño & $230: 101$ & 2.28 \\
\hline
\end{tabular}

CUADRO \#5. PREVALENCIA DE CARIES DENTAL EN ESCOLARES DE SEIS A DIEZ AÑOS

En el cuadro \# 7 se presenta los valores de Chi de las variables significativamente asociadas con la presencia de caries en dientes definitivos.

La estadística Chi es la diferencia en -2 log-de probabilidad entre el modelo final y el modelo reducido. El modelo reducido se forma omitiendo un efecto del modelo final.

El cuadro \# 8 destaca los valores de la prueba de asociación Chi de las variables significativamente vinculadas con la presencia de caries en dientes definitivos.

\section{Discusión}

En América Latina la prevalencia de caries dental es variada y depende del grupo poblacional evaluado. Así, en una zona urbana de México, Romero y Juárez (2006), la encontraron presente en $85 \%$ de la población infantil. En Bolivia, el Ministerio de Salud (2006), reportó una "prevalencia de caries de 84,6\% y el índice CPOD de 4.7 a la edad de 12 años". En Perú, Sedano (1987) estableció una prevalencia de caries dental de 94,5\% en niños de Canto Grande (zona urbanomarginal de Lima). En Costa Rica, Montero y col. (2011), en un estudio de representatividad nacional aplicado en el 2006 en escolares de 12 años, calcularon: un índice CPOS promedio de 4,1 , oscilando entre 4,88 para la Región Huetar Norte y 3,40 para la Región Central. El índice CPOD fue 2,57, y las unidades cariadas correspondieron a 39\%. La prevalencia de caries se estimó en $84,4 \%$.

Contrastando con los datos anteriores, Lorenzo (2003) en Montevideo, realizó un estudio piloto transversal, donde "casi el $50 \%$ de niños estaban libres de caries. Las escuelas de nivel socioeconómico (NSE) alto y medio se comportaban como una unidad, el porcentaje de niños libres de caries ега $60 \%$ en el nivel socioeconómico alto y en las escuelas de bajo

\begin{tabular}{|l|c|c|}
\hline Prevalencia de caries dental en escolares de once a quince años & N & $\%$ \\
\hline Total de niños de 11 a 15 años: & 212 & $100 \%$ \\
\hline Total de niños de 11 a 15 años que NO presentaron caries: & 96 & $45.3 \%$ \\
\hline Total de niños de 11 a 15 años que SI presentaron caries: & 116 & $54.7 \%$ \\
\hline Total de caries en los 116 niños: & 446 & \\
\hline Razón de caries por niño & $446: 116$ & 3.84 \\
\hline
\end{tabular}




\begin{tabular}{|l|l|r|}
\hline Variables & $\mathrm{Ch}^{2}$ & $\mathrm{p}$-Value \\
\hline Caries presente en dientes primarios & 65.692 & .000 \\
\hline Ausencia de cepillo de dientes en la escuela & 28.133 & .003 \\
\hline Baja autopercepción de su propia salud oral & 24.233 & .012 \\
\hline Ausencia/desconocimiento de una unidad de salud cercana & 21.685 & .006 \\
\hline No haber estado sano en el último mes & 21.674 & .027 \\
\hline
\end{tabular}

CUADRO \# 7. GRADO DE ASOCIACIÓN ENTRE CARIES EN DIENTES DEFINITIVOS Y VARIABLES SOCIODEMOGRÁFICAS:

nivel socioeconómico ега 11\%. El CPOD promedio fue de 1.5, variando entre 0.9 y 2.5 según el NSE".

En Ecuador las publicaciones epidemiológicas sobre salud oral son muy escasas. La Guía de Práctica Clínica sobre caries del Ministerio de Salud Pública (2015), basada en el trabajo de Raza y col. (2010), menciona que el índice CPOD de los escolares incrementa de 0,22 a los 6 y 7 años, a 2,95 a los 12 años y a 4,64 a los 15 años.

Según el estudio, realizado por Medina y col. (2008) en la Amazonia ecuatoriana, la prevalencia global de caries dental en niños indígenas ( $n=930$ ) y no indígenas ( $n=519$ ) de 6 a 12 años fue casi tan alta en ambos grupos, lo que insinuaba que probablemente ambas poblaciones habrían adoptado un tipo de dieta que ha afectado el índice CPOD entre escolares e indica la acelerada destrucción de los primeros dientes permanentes. “El valor promedio del índice CPOD para niños de 12 años de edad fue 4.47 (2.85) entre niños indígenas y 5.25 (2.89) entre niños no indígenas".

Tomando en consideración que la población indígena del Ecuador ha permanecido por centenas de años en condiciones de desventaja socioeconómica respecto del grupo social dominante, no es aventurado pensar que la presencia de caries dental podría tener algún tipo de asociación con un historial social donde la realidad indígena es distinta de la no indígena. Esta desventaja ya ha sido puesta en evidencia en estudios de pobreza, analfabetismo, acceso a servicios de salud, patrones alimentarios, desnutrición, ruralidad y etnicidad, entre otras variables. En ese sentido recomendamos revisar los trabajos de Freire WB. y col. ENSANUT ECU (2012), CEPAR, ENDEMAIN ECU (2004), el Mapa de Pobreza y Desigualdad 2008 realizado por la Coordinación de Desarrollo Social SIISE- STMCDS y otros.

\begin{tabular}{|l|l|r|}
\hline Variables & Chi $^{2}$ & p-Value \\
\hline Sexo masculino & 41.365 & .001 \\
\hline Baja autopercepción de la salud oral & 32.555 & .013 \\
\hline Última visita al dentista en más de seis meses & 28.418 & .040 \\
\hline Ausencia de cepillo en la escuela & 26.966 & .050 \\
\hline
\end{tabular}


Según Londoño y Frenk (1997), en América Latina conviene tomar en cuenta las necesidades de salud de los diferentes grupos socio económicos. El primero representa el núcleo de extrema pobreza que esta "ubicado en su mayoría en áreas rurales dispersas y zonas periurbanas marginadas" y el segundo que se encuentra en "los sectores informales que viven en la pobreza y que se ubican principalmente en áreas urbanas y periurbanas"...

En el primer caso, el acceso a los servicios de salud es mínimo o inexistente, en ocasiones esporádicas recibe campañas de salud, mientras en el segundo caso las personas tienen acceso parcial a los servicios de salud, pero no los fondos por pertenecer al sector informal de la economía y estar excluidos de la seguridad social.

Estos son los grupos a los que atienden las Brigadas Odontológicas-USFQ. El propósito de este estudio fue explorar la asociación entre determinantes sociales y prevalencia de caries dental en población de edad escolar de áreas rurales y urbanas en Ecuador. Las Brigadas Odontológicas - USFQ son una respuesta concreta a la solicitud de las comunidades o de ciertas fundaciones de ayuda social, en favor de grupos de población menos favorecidos. El servicio brindado ha sido aplicado en lugares donde las condiciones socioeconómicas son difíciles. El trabajo de diagnóstico clínico ha sido complementado por una interpretación de la realidad comunitaria donde prevalece la ruralidad, el aislamiento, escases o desconocimiento de servicios odontológicos, patrones alimentarios e higiénicos que incrementan el riesgo de caries y otra patología dental.

El criterio utilizado para el diagnóstico de caries dental a nivel de las aulas comunitarias fue el hallazgo de lesiones en las que existía una franca cavitación, con esta aclaración metodológica estamos conscientes de la posibilidad de haber introducido subdiagnóstico, ya que las lesiones incipientes o dudosas no fueron incluidas. Los resultados obtenidos reportaron una prevalencia baja de caries en dientes primarios $49 \%$ de niños, índice ceod $=2.71$ (SD 3.48), y de $47.5 \%$ en dientes definitivos con un índice CPOD promedio de 1.78, valores que contrastan con los datos del Raza y colaboradores (2010), de 2,95 a los 12 años y a 4,64 a los 15 años. La severidad de caries aparece baja probablemente porque el diagnóstico fue realizado en condiciones de brigada, es decir: con los mínimos elementos de comodidad, iluminación, instrumental y estandarización inter-observador (Kappa no establecido), y deseo de cumplir con el requisito de participar en la brigada.

Pocos son los niños que acudieron al odontólogo (27.4\%) en los últimos 6 meses, si bien no se profundizó en las razones del ausentismo, sabemos que no todas las localidades cuentan con servicios dentales y en aquellas que, si los tienen, los niños no acuden por falta de promoción de los servicios o por desconocimiento de la calidad y gratuidad del servicio. El índice ceod resultó más alto que el CPOD probablemente porque la población da menos importancia a la higiene de los dientes primarios. Esta situación se confirma con la preexistencia de caries en dientes primarios $(p<.000)$ que se asocia con la prevalencia de la lesión en dientes definitivos, reflexión también realizada por Romero y Juárez (2006).

La frecuencia del cepillado aparentemente alta (tres o más veces al día, 52.2\%) parece obedecer a un ideal-teórico de higiene dental actualmente presente en el colectivo social del país, que, sin embargo, difícilmente coincide con la realidad local pues la ausencia de cepillo en la escuela $(p<.003)$, genera un periodo largo sin posibilidad de cepillado. Los patrones higiénicos escasos se hacen evidentes cuando se constata el desconocimiento del concepto de placa bacteriana $12.3 \%$ y el de caries $40.8 \%$. Por otra parte, si bien dicen que cepillan sus dientes y que lo hacen varias veces al día, tan solo $11.8 \%$ de niños están libres de placa bacteriana. 
Otras variables que se asociaron con la presencia de caries nos hablan de una autopercepción de salud oral regular o mala $(p<.012)$, desconocimiento de la existencia de una unidad de salud cercana $(p<.006)$, y la coincidencia de un estado mórbido en el mes precedente a la brigada $(p<.027)$;

La realidad ecológica andina influye en el estado dental por la presencia de concentraciones de flúor superiores a $0.7 \mu \mathrm{g} / \mathrm{dL}$ en el agua de origen subterráneo, lo que conlleva la posibilidad de niveles nocivos que son evidenciados en la coloración de los dientes. El tiempo de exposición a altas concentraciones se detecta con mayor evidencia en niños de 13 a 15 años.

Este estudio, a diferencia del realizado por Romero y López (2006) en México, con el que no coincide, si lo hace con el trabajo de Moreno AA., y col. (2001) también de México, al establecer una asociación estadísticamente significativa entre la presencia de caries en dientes primarios con el sexo masculino de los niños examinados (Chi² 41.365; df 17; p-Value. 001), no así en niños mayores con dentición definitiva.

La demanda de asistencia odontológica en las comunidades del estudio no ha sido establecida previamente. Gracias al análisis de los índices obtenidos en este estudio podemos proponer una línea basal que permitirá a futuro medir el progreso en los esfuerzos de restauración, así, el índice de restauración en dentición primaria se encuentra en: 0.55 obturados / 3.38 CEOD x 100 = $15.8 \%$; y el índice de restauración en dentición definitiva alcanza un valor de: 0.29 obturados / 1.78 CPOD $\times 100=16.3 \%$. Comparados estos valores con el trabajo de Verdugo y colaboradores (2010), se demuestra que hay aún un esfuerzo de restauración por hacer, especialmente en dentición definitiva.

Entre las limitaciones del presente estudio debemos señalar que se detectó una prevalencia baja (<50\%) de caries dental en niños de grupos poblacionales rurales y de bajos ingresos, con dentición primaria o definitiva. Este hallazgo contrasta grandemente cuando lo comparamos con aquellos de otros países Latino Americanos (México, Bolivia, Costa Rica, Perú), cuyas prevalencias fueron superiores a $80 \%$. A diferencia de esos trabajos, los datos de este estudio fueron recolectados durante las brigadas comunitarias, realizadas por estudiantes de odontología de último año de estudios, entrenados de acuerdo con las recomendaciones de la OMS, bajo condiciones especiales de iluminación y con equipo desechable.

Por tanto, la calidad de los datos, la celeridad con que se realizó el diagnóstico, la indispensable supervisión y la correlación inter observador, no estimada, ameritan ajuste.

\section{Conclusiones}

El odontólogo profesional no puede quedar satisfecho con únicamente examinar y registrar el diagnóstico clínico de su paciente, en especial cuando la mayoría de sus pacientes consulta por una misma patología. Por el contrario, debe buscar el origen de las dolencias que afectan a la población para proponer el control de los agentes causales, indagar qué sectores de la población son los más afectados y promover la salud oral evitando la ocurrencia de casos similares.

Establecer la prevalencia de caries dental como los índices ceod y CPOD requiere un diagnóstico certero, realizado por personal estandarizado en su entrenamiento y capaz de comparar sus resultados con aquellos obtenidos por estudios de igual rigor, sin embargo, el diagnóstico odontológico cobrará mayor valor, si las condiciones socioculturales, ecológicas y económicas que rodean a la población son estimadas como factores determinantes de la salud oral.

Nuestro objetivo fue explorar la asociación entre determinantes sociales y prevalencia de caries dental en población escolar rural y urbana en 
Ecuador. Los datos de esta investigación demuestran que variables relacionadas con las condiciones socioeconómicas, culturales y ambientales al igual que el acceso a la asistencia sanitaria mantienen una asociación estadísticamente significativa con la prevalencia de caries en dientes primarios (sexo masculino, autopercepción de la salud oral, más de seis meses desde la última consulta al dentista y ausencia de cepillo dental en la escuela). De igual manera, la prevalencia de caries en dientes definitivos demostró una asociación estadísticamente significativa con: Caries presente en dientes temporales, ausencia de cepillo de dientes en la escuela; autopercepción de su propia salud oral (baja), ausencia/desconocimiento de una unidad de salud cercana, y no haber estado sano en el último mes. El análisis de los datos recogidos nos permite enriquecer el trabajo de las brigadas y de la atención odontológica en general. La investigación es una herramienta que favorece la calidad científica y académica tanto del programa de enseñanza, como del servicio a la comunidad.

Recomendaciones: El diagnóstico clínico requiere ajustes para establecer con mayor certidumbre la asociación entre caries y determinantes socioambientales.

\section{Agradecimientos}

- A todos los niños que aceptaron participar en el diagnóstico clínico y proporcionaron la información sociodemográfica durante las Brigadas Odontológicas USFQ

- A las autoridades comunitarias y escolares, así como a los padres de familia de los niños atendidos en las Brigadas Odontológicas de Servicio Comunitario de la Escuela de Odontología de la Universidad San Francisco: Escuela José Acosta Vallejo de Cangahua, Patronato San José de El Tejar, la Fundación SOYUS, el barrio Santos Pamba del sur de Quito y el proyecto Toachi Pilatón de Santo Domingo de los Tsachilas.

- A las autoridades de la Escuela de Odontología por su interés académico y científico, así como por su apoyo logístico en la realización del Servicio Comunitario e impulso al Proyecto de investigación planteado, y a la Escuela de Salud Pública de la Universidad San Francisco de Quito, por su contribución a impulsar el quehacer investigativo en odontología.

- Al Doctor José Maldonado y demás profesores de la Escuela de Odontología de la USFQ, y a todos los estudiantes que participaron en las brigadas de Servicio Comunitario 2017-2018.

- A los estudiantes Marco Carrillo, Francisco Vallejos y Pablo Baño, que colaboraron en la limpieza y transcripción de datos recolectados al programa Excel, así como en la búsqueda de información relevante sobre las comunidades. 